\title{
The association of circulating interleukin-18 with fasting insulin and weight loss in obese children
}

\author{
Tim K. Tso ${ }^{1 *}$, Wen-Nan Huang ${ }^{2}$, Chen-Kang Chang ${ }^{3}$ \\ ${ }^{1}$ Department of Food Science, National Chiayi University, 300 University Road, Chia-Yi, Taiwan, China; \\ *Corresponding Author: timtso@mail.ncyu.edu.tw \\ ${ }^{2}$ Department of Allergy, Immunology and Rheumatology, Taichung Veterans General Hospital, Taichung, Taiwan, China \\ ${ }^{3}$ Sport Science Research Center, National Taiwan Sport University, Taichung, Taiwan, China
}

Received 20 February 2010; revised 23 March 2010; accepted 25 March 2010.

\section{ABSTRACT}

Obesity is an independent risk factor for developing cardiovascular disease and increases insulin resistance in children. Interleukin (IL)-18 is a novel pro-inflammatory cytokine with potential atherogenetic properties. This study aimed to identify circulating levels of IL-18 in obese children and examine the effects of combined nutritional education-physical activity course on circulating IL-18. Plasma IL-18, body mass index (BMI), fasting glucose and insulin, homeostasis model assessment insulin resistance (HOMA IR), lipid profile, uric acid, highsensitive C-reactive protein (hs-CRP), and homocysteine were determined in 70 obese children aged 10-12 years before and after attending a 13-week weight reduction program, which included physical activities and nutritional education. Twenty-five age-matched non-obese children served as controls. At baseline, obese children had significantly higher levels of BMI, fasting insulin, HOMA IR, triglyceride (TG), uric acid, hs-CRP, and IL-18 but lower high-density lipoprotein-cholesterol (HDL-C) than non-obese children. Plasma IL-18 levels in obese children decreased significantly after the weight reduction program. At baseline, plasma IL-18 levels in obese children positively correlated with BMI, HOMA IR, insulin and TG but negatively correlated with HDL-C. There was a significant relationship between plasma IL-18 and BMI changes. Moreover, fasting insulin was responsible for IL-18 variability in obese children. These findings suggest that elevated plasma IL-18 levels in obese children are partly associated with pa- rameters of obesity and insulin resistance, and are significantly affected by modest weight loss.

Keywords: Body Mass Index; Children; Insulin; Interleukin; Obesity; Weight Loss

\section{INTRODUCTION}

The increasing prevalence of childhood obesity has significant medical and economical consequences. Obesity in the youth increases the risk for cardiovascular complications [1]. While mechanisms responsible for the increased prevalence of childhood obesity and associated chronic diseases have not been completely elucidated, its prevention and treatment play an important role in reducing cardiovascular risk. Weight loss induces decreased lipid profiles [2], insulin resistance [3], and chronic inflammatory markers [4] in obese children.

Differential low-grade inflammation is associated with obesity in the youth and some patterns of immune activation are related to insulin resistance [5]. Interleukin (IL)-18 is a novel pro-inflammatory T helper-1 cytokine produced by various cell types, including Kupffer cells, activated macrophages, keratinocytes, intestinal epithelial cells, osteoblasts, and adrenal cortex cells [6]. Recent reports indicate that IL-18 concentrations may be linked to type 2 diabetes mellitus [7,8], metabolic syndrome [9], hyperhomocysteinemia $[7,10]$, obesity $[11,12]$ and insulin resistance [12]. Weight loss and exercise training are reported to reduce IL-18 in obese adults [11,13-15]. However, the association of IL-18 with childhood obesity has not been completely established.

The purpose of the present study was to identify circulating levels of IL-18 in obese children and examine the effects of weight reduction on circulating IL-18. 


\section{MATERIALS AND METHODS}

\subsection{Subjects}

Seventy $4^{\text {th }}$-to- $6^{\text {th }}$ grade obese Chinese children (37 boys and 33 girls) aged 10-12 years from the local elementary schools were evaluated for plasma IL-18 levels and its relationship to fasting insulin and weight loss before and after completing a 13-week weight reduction program. Obesity was based on the ideal body weight (IBW) criteria and the study children were defined as obese if their body weight was greater than $120 \%$ of IBW within ageand gender-specific categories [16]. Twenty-five heightand age-matched children (13 boys and 12 girls) with body weight less than $110 \%$ of IBW from the same schools served as non-obese controls.

All of the children had similar lifestyles with no previous physical training program or dietary consults. The ethical committee of the National Taiwan Sport University (Taichung, Taiwan) approved the study and the parents of all participating children provided written informed consent.

\subsection{Research Design}

The 70 obese children participated in a 13-week weight reduction program consisting of nutritional education and physical exercise programs. Both programs were directed and supervised by dieticians, nutritionists, physicians, physical education instructors, and coaches. Both the children and their parents attended the first six lectures of the nutrition intervention program (45 minutes per lecture, two lectures per week), and the children continued the lectures thereafter.

The nutrition intervention program was taught by school dieticians and was devoted primarily to nutritional education. It included six topics: 1) introduction to the food pyramid, food categories, food and beverage choices; 2) introduction to nutrients and recommended daily nutrient intake; 3 ) understanding food preparation, balanced diet, eating habits, and over-eating control; 4) learning about dietary recall, food records, and food frequency questionnaire; 5) learning about simple concepts of the food exchange list and practice of calorie calculation; and 6) application and practice of nutrition knowledge by playing games and giving tests.

Regarding physical activity intervention, all obese children were instructed to participate in physical activeity three times per week (45 minutes per exercise session) during the study period supervised by professional youth coaches at the elementary schools. The physical activeties throughout this exercise intervention varied in intensity and duration, but were similar to the type of exercise that elementary school children regularly performed.

\subsection{Experimental Approaches}

Blood specimens before (baseline) and after (week 13) the 13-week weight reduction program were collected in the morning after an overnight fast and centrifuged immediately to obtain serum or plasma aliquots that were either used immediately or frozen at $-80^{\circ} \mathrm{C}$ until the required assay.

Fasting glucose concentration was determined using an enzymatic colorimetric method (Sigma Chemical Company, St. Louis, MO, USA), while quantitative measurement of fasting insulin concentrations was conducted using an Abbott IMx Insulin Kit based on a micro-particle enzyme immuno-assay (MEIA) (Abbott Laboratories, Dainabot, Tokyo, Japan). HOMA IR was calculated according to the formulas in the HOMA model [17]. Enzymatic methods were used to determine plasma concentrations of total cholesterol (Beckman TC Reagent) and TG (Beckman TG Reagent). Magnesium-dextran sulfate precipitation reagent was used to separate HDL-C, which was then assessed enzymatically. Low-density lipoprotein-cholesterol (LDL-C) was determined using the Friedewald equation [18].

Plasma homocysteine levels were measured using high-performance liquid chromatography with fluorescence detection. Quantitative determination of plasma hs-CRP was assessed by particle-enhanced immunonephelometry using a high sensitivity CRP immunonephelometric assay kit (Dade Behring, Marburg, Germany). Plasma IL-18 levels were assessed by enzymelinked immunosorbent assay (ELISA) using a Human IL-18 ELISA kit (Medical \& Biological Laboratories Co., LTD., Nagoya, Japan) based on the manufacturer's instructions and analyzed with a Dynex MRX II micro-plate reader (DYNEX Technologies, Inc., Chantilly, VA, USA) at a wavelength of $450 \mathrm{~nm}$. This commercial kit was used for detecting only bioactive IL-18, not the immature pro-IL-18, as has been widely used. The intraand inter-assay coefficients of variation for IL-18 measurements were $7.26 \%$ and $7.53 \%$, respectively.

\subsection{Statistical Analysis}

Tested variables for comparison of means were expressed as mean \pm standard error of mean (SEM). The distribution of tested variables was examined graphically for normality. An independent sample t test or MannWhitney $U$ test was used to examine mean differences between the obese and the non-obese children. Paired $t$ test was used to compare values obtained before and after attending the 13-week weight reduction program in all obese children. Analysis of variance (ANOVA) was used to compare values obtained from obese children stratified by BMI and fasting insulin levels. Pearson's correlation or Spearman correlation analysis was used to 
examine the relationships between IL-18 and tested variables. A stepwise multiple regression analysis was then performed to determine independent variables for plasma levels of IL-18. A $p$ value $<0.05$ was considered statistically significant. All statistical analyses were performed using the Statistical Package of Social Sciences (SPSS) 10.0 for Windows (SPSS Inc., Chicago, IL, USA).

\section{RESULTS}

The 70 obese children ( 37 boys and 33 girls) had an average age of 11 years. All completed the weight reduction program without any reported discomfort or complaints. About 67\% (47/70) of them lost weight (mean: $-1.69 \pm 0.31 \mathrm{~kg}$, range: $0.2-11 \mathrm{~kg})$ and $87 \%(61 / 70)$ had reduced BMI (mean: $-1.26 \pm 0.13 \mathrm{~kg} / \mathrm{m}^{2}$, range: 0.04-5.62 $\mathrm{kg} / \mathrm{m}^{2}$ ). In addition to reducing BMI (mean: $27.47 \pm 0.38$ vs. $\left.26.59 \pm 0.40 \mathrm{~kg} / \mathrm{m}^{2}, p<0.001\right)$, the weight reduction program also reduced plasma levels of uric acid (mean: $6.16 \pm 0.16$ vs. $5.76 \pm 0.15 \mathrm{mg} / \mathrm{dL}, p=$ 0.005 ), homocysteine (mean: $11.48 \pm 0.32$ vs. $10.06 \pm$ $0.28 \mu \mathrm{mol} / \mathrm{L}, p<0.001$ ), hs-CRP (mean: $1.18 \pm 0.02$ vs. $1.01 \pm 0.02 \mathrm{mg} / \mathrm{L}, p<0.001$ ), and IL-18 (mean: 161.44 \pm 4.03 vs. $132.57 \pm 1.48 \mathrm{pg} / \mathrm{mL}, p<0.001$ ) without significant changes in lipid profile or insulin resistance. There were no gender or age differences for weight loss and BMI reduction (data not shown).

The comparisons of test variables for the 70 obese and 25 non-obese children were shown in Table 1. Compared to non-obese children, obese children had lower HDL-C but higher levels of fasting insulin, HOMA-IR, TG, uric acid, hs-CRP, and IL-18. The plasma IL-18 levels of obese children by BMI and fasting insulin tertiles at baseline, and the effects of weight reduction were shown in Table 2. There was an increase in plasma IL-18 levels in obese children with higher BMI and fasting insulin. Obese children with fasting insulin in the middle and top tertiles had significantly higher plasma IL-18 levels than those in the bottom tertile. In addition, plasma IL-18 levels of obese children decreased significantly after the weight reduction program.

Table 3 showed the association of plasma IL-18 levels with anthropometric measures and metabolic variables in obese children at baseline. Plasma IL-18 levels in obese children at baseline positively correlated with BMI, insulin, HOMA-IR, and TG but negatively correlated with HDL-C. Moreover, there was a significant relationship between plasma IL-18 and BMI changes $(r=0.352, p=$ 0.004 ) but no significant correlations between plasma IL-18 changes and those of other tested variables after the weight reduction program (data not shown).

Moreover, stepwise multiple regression analysis was performed with plasma IL-18 as dependent variable and independent variables that included weight, BMI, fasting insulin, fasting glucose, total cholesterol, TG, LDL-C, HDL-C, uric acid, homocysteine, and hs-CRP. The results showed that fasting insulin was responsible for plasma IL-18 variability in obese children (Table 4).

\section{DISCUSSION}

The present study investigated circulating levels of IL-18 in 70 obese children and evaluated the beneficial effects of combined nutritional education-physical activity intervention. The major finding was that obese children have increased plasma IL-18 level, which is associated with BMI and markers of insulin resistance, and is improved with only modest weight loss even without improvement in insulin resistance.

Table 1. Characteristics of the study children ${ }^{\mathrm{a}}$.

\begin{tabular}{lcc}
\hline & $\begin{array}{c}\text { Non-obese } \\
\text { children }(\mathrm{n}=25)\end{array}$ & $\begin{array}{c}\text { Obese children } \\
(\mathrm{n}=70)\end{array}$ \\
\hline Gender (boy/girl) & $13 / 12$ & $37 / 33$ \\
Height $(\mathrm{cm})$ & $147 \pm 1$ & $149 \pm 1$ \\
Weight $(\mathrm{kg})$ & $43 \pm 1$ & $61 \pm 1^{*}$ \\
BMI $\left(\mathrm{kg} / \mathrm{m}^{2}\right)$ & $20.02 \pm 0.12$ & $27.47 \pm 0.38^{*}$ \\
Glucose $(\mathrm{mmol} / \mathrm{L})$ & $4.26 \pm 0.07$ & $4.28 \pm 0.06$ \\
Insulin $(\mu \mathrm{U} / \mathrm{mL})$ & $6.51 \pm 0.33$ & $20.28 \pm 1.57^{*}$ \\
HOMA IR & $1.24 \pm 0.07$ & $3.91 \pm 0.34^{*}$ \\
TC $(\mathrm{mmol} / \mathrm{L})$ & $4.16 \pm 0.13$ & $4.17 \pm 0.09$ \\
TG $(\mathrm{mmol} / \mathrm{L})$ & $0.65 \pm 0.04$ & $0.88 \pm 0.05^{*}$ \\
LDL-C (mmol/L) & $2.38 \pm 0.10$ & $2.63 \pm 0.08$ \\
HDL-C (mmol/L) & $1.48 \pm 0.05$ & $1.14 \pm 0.02^{*}$ \\
Uric acid $(\mathrm{mg} / \mathrm{dL})$ & $4.86 \pm 0.25$ & $6.16 \pm 0.16^{*}$ \\
Homocysteine $(\mu \mathrm{mol} / \mathrm{L})$ & $10.88 \pm 0.46$ & $11.48 \pm 0.32$ \\
hs-CRP $(\mathrm{mg} / \mathrm{L})$ & $0.62 \pm 0.06$ & $1.18 \pm 0.02^{*}$ \\
IL-18 $(\mathrm{pg} / \mathrm{mL})$ & $95 \pm 4$ & $161 \pm 4^{*}$ \\
\hline
\end{tabular}

Abbreviations: BMI, body mass index; HOMA IR, homeostasis model assessment insulin resistance; TC, total cholesterol; TG, triglycerides; LDL-C, low-density lipoprotein cholesterol; HDL-C, high-density lipoprotein cholesterol; hs-CRP, high sensitive C-relative protein; IL, interleukin.

${ }^{a}$ Values are expressed as mean \pm standard error of mean.

${ }^{*} p<0.05$ compared with non-obese children. 
Table 2. Plasma concentrations of IL-18 (pg/mL) by body mass index and fasting insulin tertiles at baseline, and effect of weight reduction in obese children ${ }^{\mathrm{a}}$.

\begin{tabular}{|c|c|c|}
\hline & Mean $\pm \operatorname{SEM}(95 \% \mathrm{CI})$ & $\mathrm{P}^{\mathrm{b}}$ \\
\hline \multicolumn{3}{|c|}{$\begin{array}{l}\text { Body mass index } \\
\left(\mathrm{kg} / \mathrm{m}^{2}\right) \text { tertiles }\end{array}$} \\
\hline$<25.6$ & $157 \pm 7(141-172)$ & \\
\hline $25.6-28.4$ & $173 \pm 7(159-187)$ & \\
\hline$>28.4$ & $172 \pm 6(159-185)$ & \\
\hline
\end{tabular}

0.169

Fasting insulin

$(\mu \mathrm{U} / \mathrm{mL})$ tertiles

$\begin{array}{ll}<14.8 & 151 \pm 6(138-163) \\ 14.8-21.8 & 176 \pm 9(158-194) \\ >21.8 & 176 \pm 4(168-184)\end{array}$

$>21.8-176 \pm 4(168-184)$

0.008

Weight reduction

program

Before

$161 \pm 4(114-230)$

After

$133 \pm 1(117-158)$

$<0.001$

${ }^{a}$ Values are expressed as mean \pm standard error of mean and $95 \%$ confidence interval.

${ }^{\mathrm{b}}$ Statistical significance $(p<0.05)$ of mean was determined by analysis of variance for body mass index and fasting insulin tertiles, and by paired $t$ test for effect of weight reduction.

Childhood obesity is a great concern because it is associated with many risk factors for chronic diseases later in life. Weight and BMI during childhood have been correlated positively with fasting insulin, blood pressure, and lipids in young adulthood [19]. BMI at age 13 years is highly correlated with that at age 22 years [20]. In addition, subjects with higher BMI as children show higher total cholesterol and LDL-C and insulin resistance in young adulthood [20]. The present study has found that obese children have cardiovascular risk profile in terms of lower HDL-C but higher levels of fasting insulin, HOMA-IR, TG, uric acid, hs-CRP, and IL-18 than non-obese children. After a 13-week combined nutritional education-physical activity program, $67 \%$ of obese children had lower body weight with an average weight reduction of $1.69 \mathrm{~kg}$ but range that varied from 0.2 to $11 \mathrm{~kg}$. Even though weight loss was limited, the weight reduction program not only reduced BMI but also
Table 3. Correlation coefficients of IL-18 with anthropometric measures and metabolic variables in obese children at baseline.

\begin{tabular}{lcc}
\hline & \multicolumn{2}{c}{ Obese children } \\
\cline { 2 - 3 } BMI & $\mathrm{r}$ & $\mathrm{P}$ \\
Glucose & 0.378 & $0.002^{*}$ \\
Insulin & -0.183 & 0.142 \\
HOMA IR & 0.323 & $0.008^{*}$ \\
TC & 0.248 & $0.044^{*}$ \\
TG & 0.125 & 0.316 \\
LDL-C & 0.293 & $0.017^{*}$ \\
HDL-C & 0.156 & 0.210 \\
Uric acid & -0.291 & $0.018^{*}$ \\
Homocysteine & 0.199 & 0.109 \\
hs-CRP & -0.022 & 0.858 \\
\hline
\end{tabular}

Abbreviations: BMI, body mass index; HOMA IR, homeostasis model assessment insulin resistance; TC, total cholesterol; TG, triglycerides; LDL-C, low-density lipoprotein cholesterol; HDL-C, high-density lipoprotein cholesterol; hs-CRP, high sensitive C-relative protein; IL, interleukin.

${ }^{*} p<0.05$ indicated the statistical significance.

Table 4. Stepwise multiple regression analysis in obese children at baseline ${ }^{\mathrm{a}}$.

\begin{tabular}{llll}
\hline \multicolumn{1}{c}{ Beta } & t value & P value \\
\hline $\mathrm{R}^{2}=0.143$ Adjusted $\mathrm{R}^{2}=0.115$ & & \\
$\mathrm{~F}=5.243$ & & \\
Fasting insulin & 0.287 & 2.394 & 0.008 \\
\hline
\end{tabular}

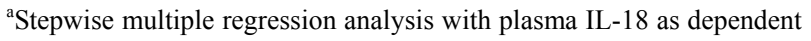
variable, while independent variables included weight, BMI, fasting insulin, fasting glucose, total cholesterol, triglyceride, LDL-C, HDL-C, uric acid, homocysteine, and hs-CRP.

lowered plasma IL-18 levels as well as other cardiovascular risk factors, such as uric acid, homocysteine, and hs-CRP.

Very few studies show an association between IL-18 and obesity in the youth, and obese children with metabolic syndrome have significantly higher IL-18 [21]. IL-18 levels are associated with BMI and waist circumference, fasting insulin, and HOMA-IR in adolescents [5]. In the present study, obese children have signifi- 
cantly higher IL-18 and such an elevation correlates with cardiovascular risk factors such as BMI, HOMA-IR, fasting insulin, TG, and HDL-C (inversely). In addition, fasting insulin explains IL-18 variability in obese children.

There are no reports that show the effects of weight loss on plasma IL-18 in obese children, but an elevation of plasma IL-18 in obese adults can be influenced by interventions related to weight control. Plasma IL-18 decreases significantly after a 12-month weight loss program with energy restrictions in obese women [11]. A 15-week hypo-caloric diet and daily exercise program reduces body weight, plasma IL-18, and increases insulin sensitivity in obese adults [22], while massive weight loss induced by gastric bypass reduces plasma IL-18 [14]. A multi-disciplinary weight reduction program emphasizing lifestyle changes is associated with reduced IL-18 in obese pre-menopausal women [23]. An 8-week exercise training also reduces adipose tissue IL-18 mRNA content in obese adults [15]. In the present study, a 13-week combined nutritional education-physical activity program has reduced plasma IL-18 without significant changes in insulin sensitivity in obese children. After the weight reduction program, $87 \%$ of obese children in the present study have shown reduced BMI, but only $41 \%$ show decreased HOMA IR and about $43 \%$ of BMI-reduced obese children show decreased HOMA IR. Taken together, a decrease in plasma IL-18 caused by the weight reduction program in obese children is at least, in part, due to changes of BMI per se, regardless of the status of insulin resistance.

Systemic inflammation has been demonstrated in overweight children as indicated by elevated CRP levels [24]. It has also been shown that obese children have higher hs-CRP levels than non-obese children [4,25] and weight loss is associated with a significant decrease in hs-CRP levels [4]. Homocysteine concentration is significantly higher in hyper-insulinemic obese children than in the normo-insulinemic obese children [25]. Homocysteine levels are significantly lower after a 12-week weight loss program with dietary advice and light exercise in obese middle-aged women with high homocysteine levels at baseline [26]. In the present study, plasma levels of hs-CRP and homocysteine in obese children have decreased significantly after the weight reduction program. However, there is no relationship among hs-CRP, homocysteine, and IL-18 before and after the weight reduction program. The mechanism associated with elevated circulating IL-18 and the effect of weight loss on IL-18 in obese children seems to be independent from other cardiovascular risk factors, such as CRP and homocysteine, and warrants further elucidation.

The strength of the present study includes the effects of the combined nutritional education-physical activity intervention on obesity-related and/or obesity-independent parameters in obese children, particularly IL-18, which has been better studied in obese adults. However, there are some weakness and limitations. Individual or synergistic effects of nutritional education and exercise on weight changes are not distinguished. In addition, there is no full quantification of the interventions accomplished by the subjects due to lack of dietary records, calorie counts, and estimates of physical activity intensity. Thus, the cause and effect in the relationship between weight loss and improvements of tested variables remain to be elucidated.

In conclusion, there are elevated circulating IL-18 levels in obese children. Modest weight loss through weight reduction intervention has the beneficial effect of reducing IL-18.

\section{ACKNOWLEDGEMENTS}

This study was supported by a research grant from the National Science Council of Taiwan (NSC 95-2413-H-415-015). The authors wish to thank Ms Zih-Chun Lin for her technical assistance.

\section{REFERENCES}

[1] Ho, T.F. (2009) Cardiovascular risks associated with obesity in children and adolescents. Annals Academy of Medicine Singapore, 38(1), 48-56.

[2] Nemet, D., Barkan, S., Epstein, Y., Friedland, O., Kowen, G. and Eliakim, A. (2005) Short-and long-term beneficial effects of a combined dietary-behavioral-physical activity intervention for the treatment of childhood obesity. Pediatrics, 115(4), e443-e449.

[3] Reinehr, T., Kiess, W., Kapellen, T. and Andler, W. (2004) Insulin sensitivity among obese children and adolescents, according to degree of weight loss. Pediatrics, 114(6), 1569-1573.

[4] Reinehr, T., Stoffel-Wagner, B., Roth, C.L. and Andler, W. (2005) High-sensitive C-reactive protein, tumor necrosis factor alpha, and cardiovascular risk factors before and after weight loss in obese children. Metabolism, 54(9), $1155-1161$.

[5] Herder, C., Schneitler, S., Rathmann, W., Haastert, B., Schneitler, H., Winkler, H., Bredahl, R., Hahnloser, E. and Martin, S. (2007) Low-grade inflammation, obesity and insulin resistance in adolescents. The Journal of Clinical Endocrinology \& Metabolism, 92(12), 45694574.

[6] Dinarello, C.A. (1999) IL-18: A Th-1-inducing, pro-inflammatory cytokine and new member of the IL-1 family. Journal of Allergy and Clinical Immunology, 103(1), 11-24.

[7] Aso, Y., Okumura, K., Takebayashi, K., Wakabayashi, S. and Inukai, T. (2003) Relationships of plasma interleukin-18 concentrations to hyperhomocysteinemia and carotid intimal-media wall thickness in patients with type 
2 diabetes. Diabetes Care, 26(9), 2622-2627.

[8] Esposito, K., Nappo, F., Giugliano, F., Di Palo, C., Ciotola, M., Barbieri, M., Paolisso, G. and Giugliano, D. (2003) Cytokine milieu tends toward inflammation in type 2 diabetes. Diabetes Care, 26(5), 1647.

[9] Hung, J., McQuillan, B.M., Chapman, C.M.L., Thompson, P.L. and Beilby, J.P. (2005) Elevated interleukin-18 levels are associated with the metabolic syndrome independent of obesity and insulin resistance. Arteriosclerosis, Thrombosis, and Vascular Biology, 25(6), 1268-1273.

[10] McLachlan, C.S., Chua, W.C., Wong, P.T., Kah, T.L., Chen, C. and El Oakley, R.M. (2005) Homocysteine is positively associated with cytokine IL-18 plasma levels in coronary artery bypass surgery patients. Biofactors, 23(2), 69-73.

[11] Esposito, K., Pontillo, A., Ciotola, M., Di Palo, C., Grella, E., Nicoletti, G. and Giugliano, D. (2002) Weight loss reduces interleukin-18 levels in obese women. The Journal of Clinical Endocrinology \& Metabolism, 87(8), 3864-3866.

[12] Escobar-Morreale, H.F., Botella-Carretero, J.I., Villuendas, G., Sancho, J. and San Millan, J.L. (2004) Serum interleukin-18 concentrations are increased in the polycystic ovary syndrome: Relationship to insulin resistance and to obesity. The Journal of Clinical Endocrinology \& Metabolism, 89(2), 806-811.

[13] Madsen, E.L., Bruun, J.M., Skogstrand, K., Hougaard, D.M., Christiansen, T. and Richelsen, B. (2009) Longterm weight loss decreases the nontraditional cardiovascular risk factors interleukin-18 and matrix metalloproteinase-9 in obese subjects. Metabolism, 58(7), 946-953.

[14] Vilarrasa, N., Vendrell, J., Sanchez-Santos, R., Broch, M., Megia, A., Masdevall, C., Gomez, N., Soler, J., Pujol, J., Bettonica, C., Aranda, H. and Gomez, J.M. (2007) Effect of weight loss induced by gastric bypass on proinflammatory interleukin-18, soluble tumor necrosis factor-alpha receptors, C-reactive protein and adiponectin in morbidly obese patients. Clinical Endocrinology, 67(5), 679-686.

[15] Leick, L., Lindegaard, B., Stensvold, D., Plomgaard, P., Saltin, B. and Pilegaard, H. (2007) Adipose tissue interleukin-18 mRNA and plasma interleukin-18: Effect of obesity and exercise. Obesity, 15(2), 356-363.

[16] Chu, N.F. (2001) Prevalence and trends of obesity among school children in Taiwan-the Taipei Children Heart Study. International Journal of Obesity, 25(2), 170-176.

[17] Matthews, D.R., Hosker, J.P., Rudenski, A.S., Naylor,
B.A., Treacher, D.F. and Turner, R.L. (1985) Homeostasis model assessment: Insulin resistance and $\beta$-cell function from fasting plasma glucose and insulin concentrations in man. Diabetologia, 28(7), 412-419.

[18] Friedewald, W.T., Levy, R.I. and Fredrickson, D.S. (1972) Estimation of the concentration of low-density lipoprotein cholesterol in plasma without use of the preparative ultra-centrifuge. Clinical Chemistry, 18(6), 499-502.

[19] Sinaiko, A.R., Donahue, R.P., Jacobs, D.R.Jr. and Prineas, R.J. (1999) Relation of weight and rate of increase in weight during childhood and adolescence to body size, blood pressure, fasting insulin, and lipids in young adults. The Minneapolis Children's Blood Pressure Study. Circulation, 99(11), 1471-1476.

[20] Steinberger, J., Moran, A., Hong, C.P., Jacobs, D.R. Jr. and Sinaiko. A.R. (2001) Adiposity in childhood predicts obesity and insulin resistance in young adulthood. Journal of Pediatrics, 138(4), 469-473.

[21] Gilardini, L., McTernan, P.G., Girola, A., da Silva, N.F., Alberti, L., Kumar, S. and Invitti, C. (2006) Adiponectin is a candidate marker of metabolic syndrome in obese children and adolescents. Atherosclerosis, 189, 401-407.

[22] Bruun, J.M., Stallknecht, B., Helge, J.W. and Richelsen, B. (2007) Interleukin-18 in plasma and adipose tissue: Effects of obesity, insulin resistance, and weight loss. European Journal of Endocrinology, 157(4), 465-471.

[23] Esposito, K., Pontillo, A., Di Palo, C., Giugliano, G., Masella, M., Marfella, R. and Giugliano, D. (2003) Effect of weight loss and lifestyle changes on vascular inflammatory markers in obese women: A randomized trial. The Journal of the American Medical Association, 289(14), 1799-1804.

[24] Visser, M., Bouter, L.M., McQuillan, G.M., Wener, M.H. and Harris, T.B. (2001) Low-grade systemic inflammation in overweight children. Pediatrics, 107(1), E13.

[25] Martos, R., Valle, M., Morales, R., Canete, R., Gavilan, M.I. and Sanchez-Margalet, V. (2006) Hyperhomocysteinemia correlates with insulin resistance and low-grade systemic inflammation in obese pre-pubertal children. Metabolism, 55(1), 72-77.

[26] Sheu, W.H., Chin, H.M., Lee, W.J., Wan, C.J., Su, H.Y. and Lang, H.F. (2005) Prospective evaluation of folic acid supplementation on plasma homocysteine concentrations during weight reduction: A randomized, double-blinded, placebo-controlled study in obese women. Life Sciences, 76(18), 2137-2145. 